\title{
Supplementation of triple viable probiotics combined with dietary intervention is associated with gut microbial improvement in humans on a high-fat diet
}

\author{
LEIMIN QIAN $^{1}$, RENYUAN GAO $^{2}$, JIANMING HUANG $^{1}$ and HUANLONG QIN ${ }^{2}$ \\ ${ }^{1}$ Department of Gastrointestinal Surgery, Jiangyin People's Hospital, Jiangyin, Jiangsu 214400; \\ ${ }^{2}$ Department of General Surgery, The Tenth People's Hospital Affiliated to Tongji University, Shanghai 200072, P.R. China
}

Received December 21, 2018; Accepted June 20, 2019

DOI: $10.3892 /$ etm.2019.7801

\begin{abstract}
Numerous animal studies have demonstrated that oral probiotics may have a beneficial role in preventing obesity, inflammatory bowel disease and even colorectal cancer, which are all associated with a high-fat diet (HFD). However, the underlying beneficial effects of combined probiotic and dietary intervention on the gut microbiota of 'non-patient' individuals previously on an HFD have yet to be fully elucidated. In the present study, fecal samples were obtained from 36 volunteers on a high-fat diet and after dietary intervention for 4 months, and 16S rDNA sequencing was applied to identify how probiotics and dietary intervention had altered the composition of the microbiota. The results demonstrated that probiotics treatment and dietary intervention in combination raised the diversity of lumen microbes compared with their individual applications. A markedly separated distribution ( $\beta$-diversity) was observed, confirming the difference in gut microbiota composition among the treatment groups. Bacterial taxonomic analysis demonstrated that the relative abundance of 30 species was altered among the groups following dietary intervention and/or probiotic supplementation. The majority of the species that exhibited a population increase belonged to two butyrate-producing families, Ruminococcaceae and Lachnospiraceae, whereas the species with reduced populations mainly belonged to the Bacteroidaceae family. Collectively, these results suggest that combined probiotic and dietary intervention is able to improve the gut microbiota composition of human subjects on an HFD.
\end{abstract}

Correspondence to: Professor Huanlong Qin, Department of General Surgery, The Tenth People's Hospital Affiliated to Tongji University, 301 Yanchang Middle Road, Zhabei, Shanghai 200072, P.R. China

E-mail: huanlong_qin@live.cn

Key words: high-fat diet, gut microbiota, probiotics, dietary intervention, community population

\section{Introduction}

Approximately $10^{3}$ distinct bacterial species, the total number of which is $>10$-fold that of eukaryotic cells, colonize in the human gastrointestinal tract $(1,2)$. The commensal intestinal microbiota fulfills important roles in digestion and absorption of nutrients, the composition of the gut mucosal barrier, host metabolism and the innate immune system (3-7). A healthy gut microbiota maintains homeostasis within the host, which may be crucial to the normal operation of other vital organs, including the liver (8-11) and the brain (12-15). Accumulating evidence suggests that long-term dietary habits may result in various gut microbiota, comprising different bacterial ecosystems, which are known as enterotypes (16-18). A Western-style diet, high in total and saturated fat, is relevant to the prevalence of numerous illnesses and conditions, including obesity, type 2 diabetes, inflammatory bowel disease (IBD) and digestive tract cancers, probably due to an imbalance in gut microbial populations. A study by Kim et al (19), which revealed that a high-fat diet (HFD) induced low-grade inflammation, demonstrated the pro-inflammatory effects of energy-dense diets on vagal gutbrain communication. David et al (20) reported that increases in the growth of the sulfite-reducing pathobiont Bilophila wadsworthia in the presence of a diet rich in animal fats supported an association between dietary fat, bile acids and the outgrowth of microbes, which was able to trigger IBD. Schulz et al (21) demonstrated that an HFD accelerated tumor progression in the small intestine of K-ras ${ }^{\text {G12Dint }}$ mice, which was independent of the occurrence of obesity. Ou et al (22) reported that the effects of dietary fat on the occurrence of colorectal cancer (CRC) may be indirectly mediated by secondary bile acids, which are produced via the enterobacterial $7 \alpha$-dehydroxylation of primary bile acids and are considered to be potential carcinogens in the etiology of CRC. However, a direct association between diet, the intestinal microbiota and the pathogenesis of CRC has yet to be conclusively demonstrated, even in animal models. Of note, a number of epidemiological studies have demonstrated that excessive intake of animal fat is associated with an increased risk of colon cancer, which may promote the expansion of sulfate-reducing bacteria that are thereby able to produce genotoxic agents, including hydrogen sulfide. 
Probiotics consist of live beneficial bacterial species for promoting health of the host, typically lactobacilli and bifidobacteria, and these directly improve the composition of the colonic microbiota (23). A study by $\mathrm{Hu}$ et al (24) that employed a high cholesterol diet-fed rat model suggested that two Lactobacillus strains exerted a positive effect on lipid metabolism. Lactobacillus delbrueckii and Bifidobacterium animalis var. lactis administered alone were indicated to ameliorate colonic preneoplastic lesions in mice, although their administration in combination did not prove to be effective (25). Bertkova et al (26) demonstrated that probiotic Lactobacillus plantarum together with bioactive compounds was able to suppress colon carcinogenesis in $\mathrm{N}, \mathrm{N}$-dimethylhydrazine-induced rats. In China, capsules containing live, combined Lactobacillus, Bifidobacterium and Enterococcus have been widely used as probiotics in clinical settings, including the treatment of non-alcoholic fatty liver disease (27), irritable bowel syndrome (28) and even gastrointestinal tumors $(29,30)$, and these probably work via reducing the inflammatory response and forming a biological protective barrier.

Various functional effects of different probiotic strains on the gut microbiota and relevant diseases have been clearly demonstrated. However, certain studies have identified that gut microbiota undergo a less pronounced response to oral probiotics, partly due to inter-individual variation in microbiota composition elicited by dietary habits, in addition to the genetic background, age, other environmental factors, or the application of different analytical methods. In this regard, the concept of three enterotypes was raised by Arumugam et al (16), namely Bacteroides, Prevotella and Ruminococcus. In reality, it was difficult to categorize human beings as a particular 'enterotype', since dietary factors may have an impact on gut microbial populations $(31,32)$. A previous study by our group demonstrated a different composition of stool bacterial genera when comparing between individuals on either a high- or a low-fat diet (33). Since diet may shape the composition and function of the gut microbiota, in turn influencing host health, one promising therapeutic strategy aimed at improving health would be to alter the gut microbiome using dietary intervention.

Although the positive effects of probiotics and dietary intervention on IBD and CRC have been broadly demonstrated, comparative studies to identify the efficacy of probiotic supplementation and dietary intervention as prophylactic tools previously under HFD conditions require further investigation ahead of the occurrence of gastrointestinal diseases. Therefore, the present study aimed to assess whether probiotic treatment and dietary intervention exert any impact on the gut microbiota in human subjects on an HFD.

\section{Subjects and methods}

Subjects, diets and experimental design. A total of 36 healthy volunteers (age, 45-65 years; males/females, 16/20) from Zhouzhuang Town (Jiangyin City, China), who were consuming an HFD with dietary fat accounted for $>40 \%$ of total energy (34), were enrolled mainly according to the results of questionnaire and physical examination during the community health survey. All experiments of the present study were approved by the Ethics Committee of Shanghai
Tenth People's Hospital (Shanghai, China) and Jiangyin People's Hospital (Jiangyin, China). Written informed consent was obtained from all of the participants prior to their enrolment. Subsequently, these volunteers were randomly assigned to four groups: i) The HFD group, where the HFD was maintained due to their constant habits to establish a control group ( $n=9)$; ii) the dietary intervention (DI) group, where the HFD was replaced by a low-fat diet (LFD) in which dietary fat accounted for $<40 \%$ of total energy $(n=9)$; iii) an HFD + Probiotic group, where the HFD was supplemented with a daily dose of $2 \mathrm{~g}$ live combined Lactobacillus acidophilus $\left[\geq 1.0 \times 10^{7}\right.$ colony-forming units $\left.(\mathrm{Cfu}) / \mathrm{g}\right]$, Bifidobacterium longum $\left(\geq 1.0 \times 10^{7} \mathrm{Cfu} / \mathrm{g}\right)$ and Enterococcus faecalis $\left(\geq 1.0 \times 10^{7} \mathrm{Cfu} / \mathrm{g}\right.$ ) powder (Shanghai Xinyi Pharmaceutical Co., Ltd.) administered orally in water according to the manufacturer's recommendation $(n=9)$; and iv) the DI + Probiotic group, which received a combination of the LFD and the above-mentioned probiotic microorganisms $(n=9)$. No significant differences in daily intake of dietary fiber, or the percentages of calories derived from fat, were identified among the four groups prior to the experiment (Fig. 1). The volunteers also had a similar gender ratio, were of a similar age and body mass index, yielded similar valid sequencing reads for fecal microbiota and had similar gut microbiota compositions at the start of the experiment. These details were presented in Supplementary Table SI. Probiotic treatment and dietary intervention were continued for 4 months (from Jan 1st, 2015 to Apr 30th, 2015), which hopefully allowed sufficient duration for intestinal flora change. All of the volunteers completed the study under strict quality control with dietary changes and medication compliance monitored throughout the study.

Stool collection. A freshly voided stool sample was collected from each subject at the end of the experiment (Apr 30th, 2015), and was snap-frozen in liquid nitrogen. All samples were finally transferred to a deep freezer at $-80^{\circ} \mathrm{C}$ until the microbiota was analyzed.

\section{Microbiota analysis}

DNA extraction. Metagenomic DNA was extracted from each fecal sample using a MicroElute Genomic DNA kit (cat. no. D3096-01; Omega; BioTek, Inc.) according to the manufacturer's protocol. The MicroElute Genomic DNA kit extraction controls were included through the DNA extraction and PCR steps as a negative control. Total DNA was eluted in $50 \mu 1$ elution buffer using a method modified from the manufacturer's protocol (Qiagen; GE Healthcare) and stored at $-20^{\circ} \mathrm{C}$ prior to performing qPCR.

PCR amplification and 16S rDNA sequencing. The primers $319 \mathrm{~F}$ and $806 \mathrm{R}$ were used to amplify the double hypervariable $\mathrm{V} 3$ and V4 regions of the bacterial 16S rRNA. PCR procedures were as follows: Initial denaturation $\left(30 \mathrm{sec}\right.$ at $\left.98^{\circ} \mathrm{C}\right)$, followed by 35 cycles of amplification including denaturation $(10 \mathrm{sec}$ at $\left.98^{\circ} \mathrm{C}\right)$, annealing $\left(30 \mathrm{sec}\right.$ at $\left.54^{\circ} \mathrm{C}\right)$ and extension $\left(45 \mathrm{sec}\right.$ at $\left.72^{\circ} \mathrm{C}\right)$, and then final extension $\left(10 \mathrm{~min}\right.$ at $\left.72^{\circ} \mathrm{C}\right)$. The PCR products for each example were normalized using an AxyPrep ${ }^{\circledR}$ TM Mag PCR Normalizer (Axygen Biosciences), followed by purification with AMPure XT beads (Beckman Coulter Genomics), 


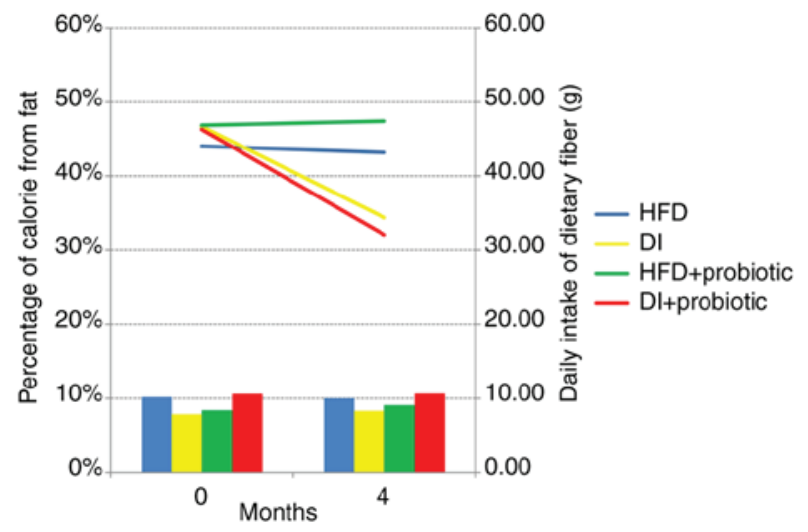

Figure 1. Composition of experimental diet. Dietary fat of the HFD accounted for $>40 \%$ of total energy, whereas that of the DI was $<40 \%$. Furthermore, no significant changes in the daily intake of dietary fiber in any of the groups occurred between day 0 and the end of the experiment (at month 4). HFD, high-fat diet; DI, dietary intervention with low-fat diet.

and quantification using the Illumina Library Quantification kit (Kapa Biosciences). Pyrosequencing was performed using a MiSeq System (Illumina, Inc.).

Bioinformatics analysis. After filtering the raw data, the high-quality sequences were identified, which were subsequently clustered into operational taxonomic units (OTUs) using the CD-hit-est-based clustering method (35). The numbers of the sequences and OTUs for each sample were calculated by using the software PyNAST (http:/qiime. org/pynast/). Subsequently, analysis of the $\alpha$-diversity (i.e., the mean species diversity in sites or habitats at a local scale), including the Observed species, Shannon, Simpson and Chaol indexes, was performed. A Venn diagram of the common and unique OTUs among the four groups was constructed using online software (http://bioinfogp.cnb.csic.es/tools/venny/). Unweighted Unifrac distance metrics analysis and principal coordinate analysis (PCoA) were also performed in terms of the matrix of distance to assess the $\beta$-diversity (i.e., the ratio between regional and local species diversity). The relative abundance (\%) of bacteria at the phylum and species taxa levels in each sample were calculated by using RDP-derived taxonomic communities. A heatmap on species information was constructed using Heml software (version 1.0.3.3) (36).

Statistical analysis. Student's t-test and Kruskal-Wallis one-way analysis of variance with Bonferroni's correction control were performed using SPSS version 19.0 (IBM Corp.). $\mathrm{P}<0.05$ was considered to indicate a statistically significant difference.

\section{Results}

Effects of dietary intervention and oral probiotics on the gut microbial diversity indices in an HFD population. A total of $2,203,533$ pyrosequencing reads for the fecal microbiota were analyzed with a mean of 63,573,57,697,63,598 and 59,968 reads for the HFD, DI, HFD + Probiotic and DI + Probiotic group, respectively. According to the indices of Observed species, Shannon and Chaol for stool (Fig. S1), but not according to the Simpson index, the adiversity of the fecal microbiota of the DI + Probiotic group was higher compared with that of the DI group or the HFD group $(\mathrm{P}<0.05)$. However, there was no significant difference between the HFD and the DI group, or between the HFD + Probiotic and the DI + Probiotic group. $\beta$-diversity analysis revealed that the fecal samples were distributed diffusely and apparent non-overlapping clusters among groups were also formed (Fig. 2).

Effects of dietary intervention and oral probiotics on the gut microbiota composition of an HFD population. The three most abundant phyla in the fecal microbionta, including Firmicutes, Bacteroidetes and Proteobacteria, were not significantly different among the groups at 4 months (Fig. 3A). The relative abundance of the other phyla (mainly including Actinobacteria and Fusobacteria) was low in all four groups. There was no apparent inter-individual variability at the phylum level within each group (Fig. 3B).

Subsequently, the differences in shared species among groups were analyzed. Overall, there were 467 species detected in stool samples $(323,385,411$ and 418 species in the HFD, DI, HFD + Probiotic and DI + Probiotic group, respectively). When comparing 100\% (9/9), 89\% (8/9), 78\% (7/9), 67\% (6/9), 56\% (5/9), 44\% (4/9) and 11\% (1/9) of the populations in each group with each other, the number of species shared among all groups was 5, 9, 22, 43, 74, 109 and 270, respectively. These results suggested that the number of bacterial species shared between the groups is dependent on how many subjects in each group were used for the comparison between the groups (Fig. S2).

Subsequently, the differences in species composition of the fecal microbiota were analyzed. In total, 30 species were different among the four groups, and the majority of these were unclassified with the exception of two distinct species, Prevotella copri and Bacteroides ovatus. It was revealed that 27 species were altered in subjects receiving dietary intervention and probiotic treatment compared with the other three groups. The relative abundance of 5, 9 and 25 species in the DI, HFD + Probiotic and DI + Probiotic groups, respectively, was significantly different compared with the HFD group $(\mathrm{P}<0.05$; Fig. 4). The relative abundance of 17 species, predominantly belonging to the Ruminococcaceae and Lachnospiraceae families of the order Clostridiales and the phylum Firmicutes, was increased in subjects from the DI + Probiotic group compared with the HFD group, whereas 8 species were decreased in the DI + Probiotic group, particularly two Bacteroides species with relatively higher abundance (B. ovatus and one unclassified) belonging to the Bacteroidaceae family of the order Bacteroidales and the phylum Bacteroidetes. Other reduced unclassified species associated with probiotic supplementation and dietary intervention belonged to various different bacterial families: Flavonifractor, Clostridium XVIII, Veillonella, Anaerostipes and Fusobacterium.

\section{Discussion}

In the present study, healthy human subjects who were consuming an HFD were selected as research subjects rather than rodents, e.g. mice or rats, whose confounding factors, including genetic background, age, sex and diet, may be well controlled, and also in preference to human subjects who had 
A

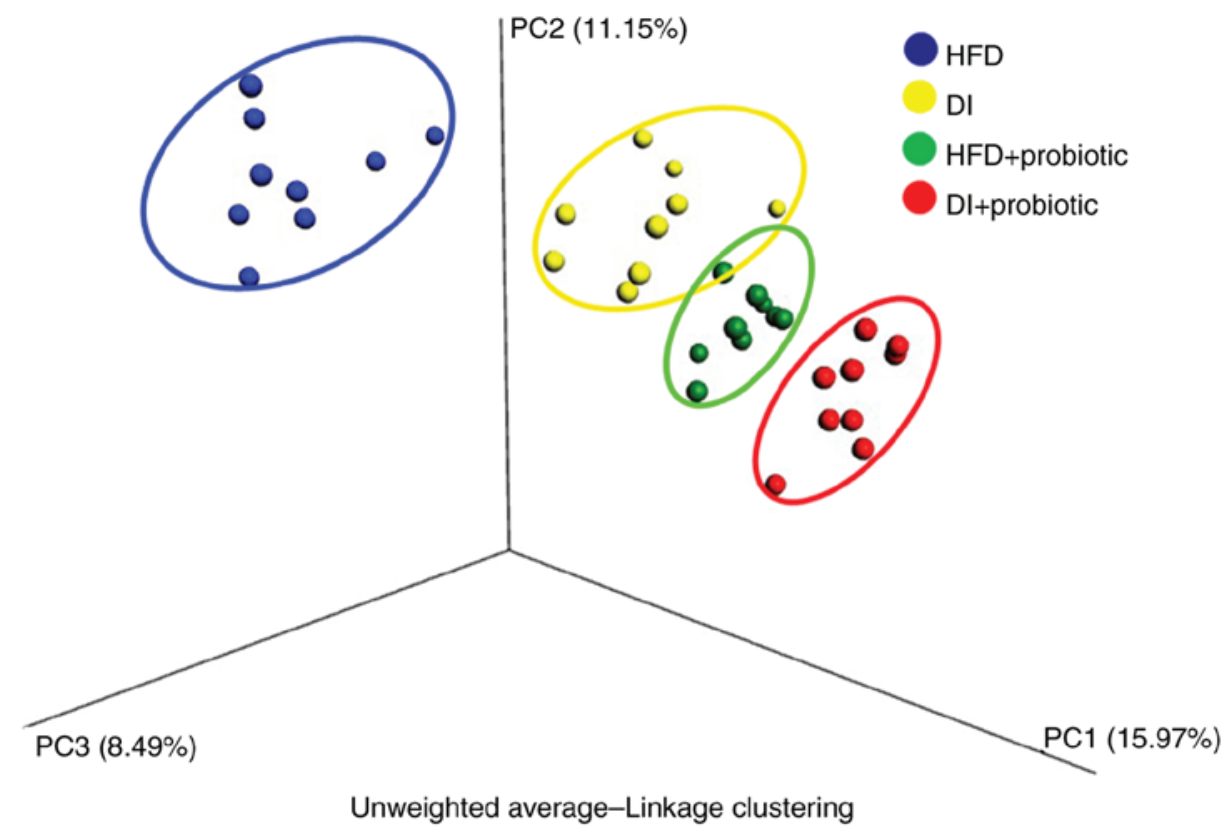

$\mathrm{B}$

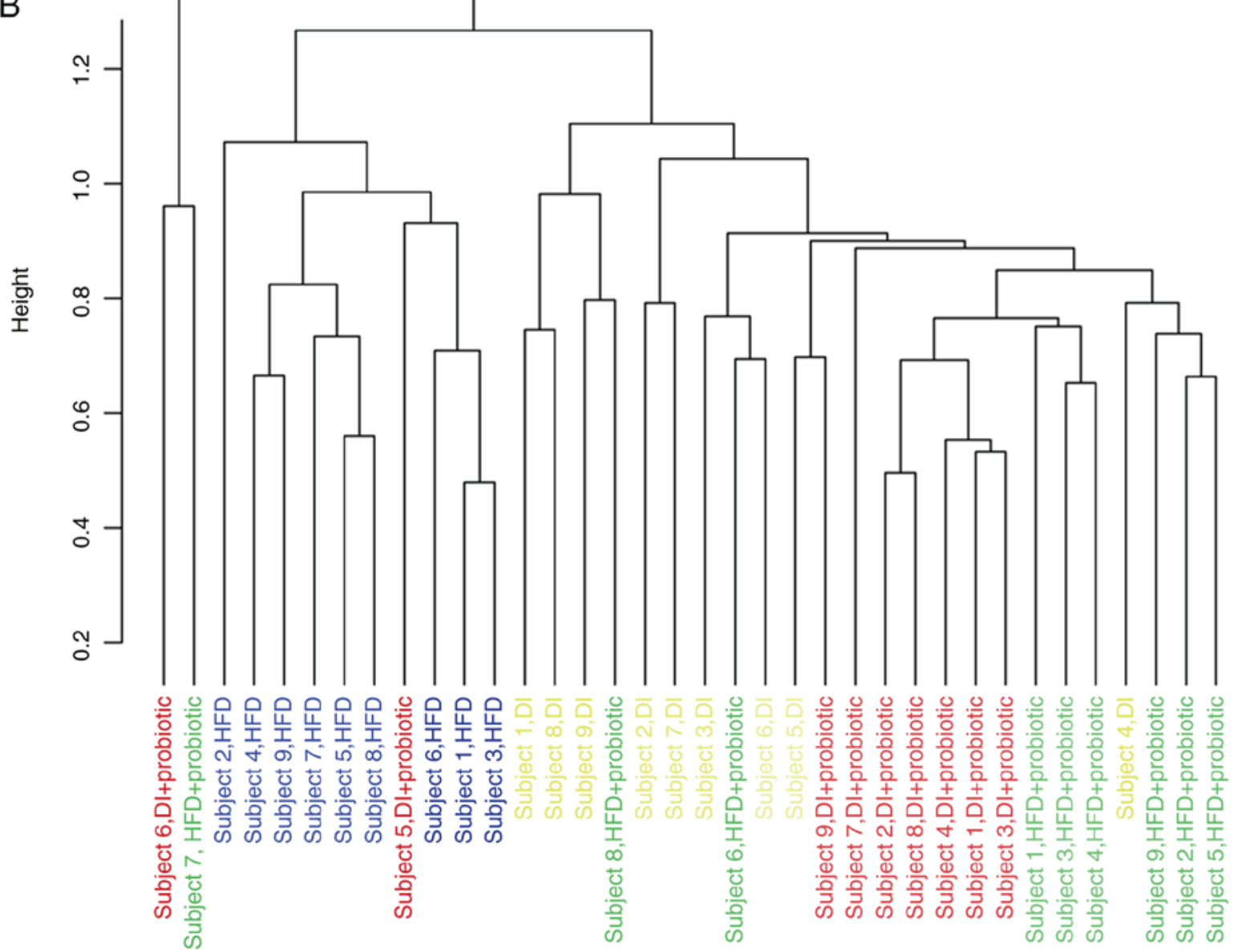

Figure 2. Clustering of samples based on fecal microbiota communities. (A) Principal coordinates analysis of samples from the HFD, DI, HFD + Probiotic and DI + Probiotic groups. (B) Unweighted pairgroup method with arithmetic mean of samples from the HFD, DI, HFD + Probiotic and DI + probiotic groups. Probiotics included Bifidobacterium longum, Lactobacillus acidophilus and Enterococcus faecalis. PC1, PC2 and PC3, three eigenvalues calculated by distance matrix of fecal samples, represent the top three principal coordinate components explaining as much of the variability in the data as possible. HFD, high-fat diet; DI, dietary intervention with low-fat diet.

already incurred disease entities, including obesity, type 2 diabetes and intestinal disorders. Furthermore, under clinical conditions, not all individuals on an HFD develop obesity, hyperlipemia, IBD and intestinal tumors, as other factors are involved, including genetic background, dietary pattern, energy expenditure, metabolic capability and intestinal barrier 
A Comparison of the average relative abundance at phylum level
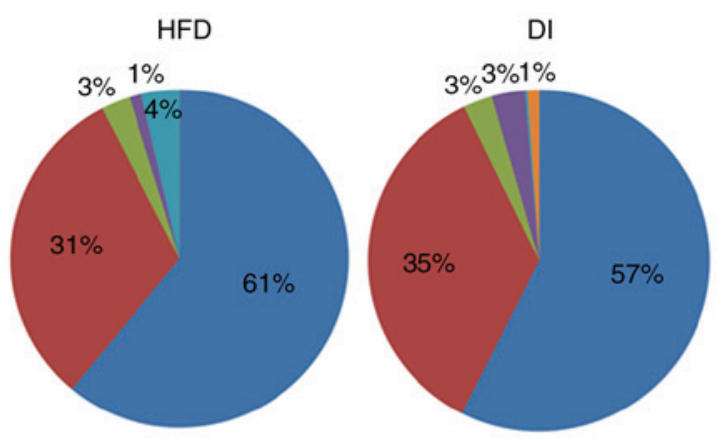
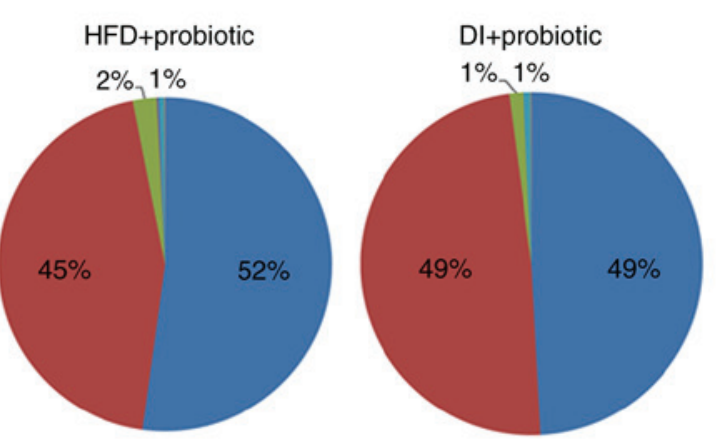

- Bacteroidetes

- Firmicutes

- Proteobacteria

- Fusobacteria

- Actinobacteria

- Others

B Inter-individual variability at phylum level

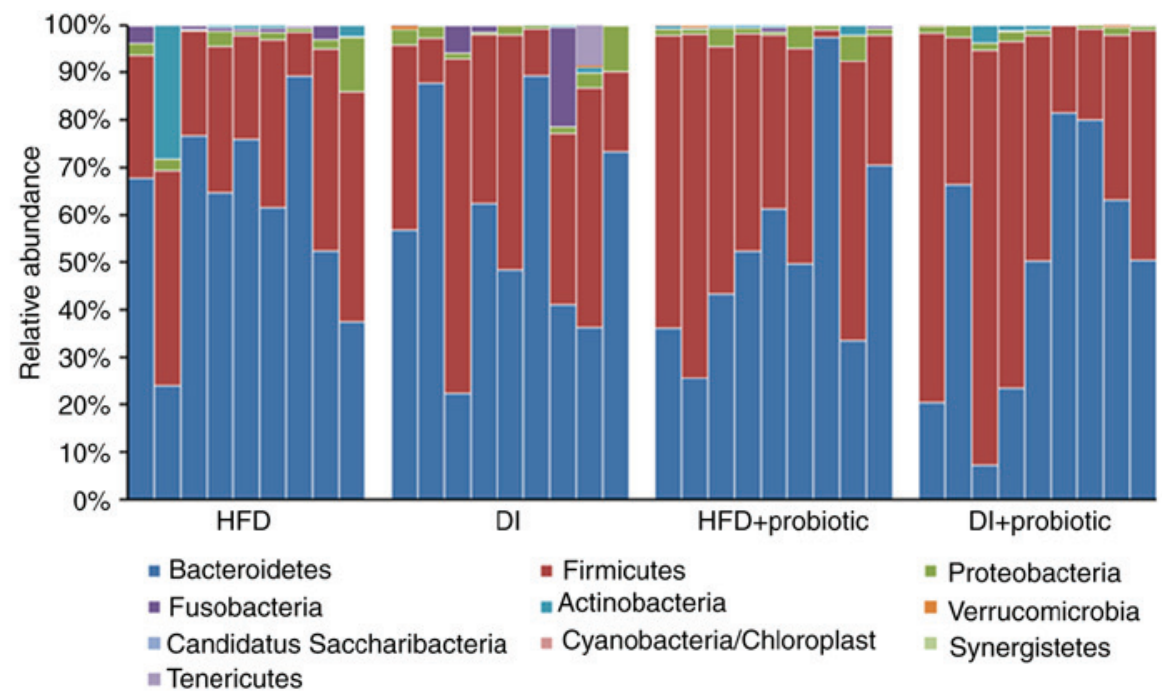

Figure 3. Effect of dietary intervention and probiotic supplementation on the fecal microbiota composition at the phylum level. (A) Pie charts displaying the average relative abundance of stool phyla. (B) Bar graphs indicating the inter-individual variability of stool bacteria at the phylum level. HFD, high-fat diet; DI, dietary intervention with low-fat diet.

state. Although a large number of reviews have described how probiotics are regarded as a gut microbiota-targeted therapy to treat HFD-induced obesity, type-2 diabetes and gastrointestinal diseases $(23,37-39)$, the role of combined oral probiotics and dietary intervention in the modulation of the gut microbiota of healthy populations consuming an HFD remained to be fully elucidated. The results of the present study indicated that probiotics may provide a means of improving the gut microbiota of HFD populations naturally, and that this effect may be enhanced by combining probiotics with dietary intervention. To a certain extent, the gut microbiota may be a suitable target of therapeutic intervention to prevent those individuals on an HFD from developing the above-mentioned diseases.

First, the present results indicated that the diversity of gut microbiota of individuals receiving an HFD may be altered after probiotic supplementation with three live bacterial strains and dietary intervention. Probiotic supplementation in two groups led to a more distinct microbial clustering that was closer in the PCoA plot, irrespective of dietary patterns. This combined effect on adults on an HFD has been rarely reported. However, the topic of changes in the diversity of the gut microbiota remains controversial (40). It was found that daily consumption of yogurt including lactobacilli and bifidobacteria by healthy medical students increased the $\alpha$-diversity of the intestinal microbiome (41). However, other studies were unable to identify any significant changes in gut microbial diversity, either on the basis of terminal restriction fragments or next-generation sequencing analysis, when comparing day 0 and day 42 of yogurt consumption (42). Therefore, the overall composition of the gut microbial community and its diversity may be governed and affected by the employed detection method of the bacteria. On the other hand, a systematic review of randomized controlled trials were observed no effects of probiotics on the fecal microbiota composition in terms of $\alpha$-diversity in any of the included studies when compared with a placebo, whereas only one study identified that probiotics were able to significantly modify the fecal bacterial community in terms of $\beta$-diversity (43). The consumption of Lactobacillus casei Zhang et al (44) markedly altered the composition of the intestinal microbiota and the gut microbiota diversity. Park et al (45), also demonstrated that mice in a probiotic treatment group had a lower gut microbiota diversity. Although low gut microbiota diversity is usually a hallmark of intestinal dysbiosis, factors including ecological stability, idealized composition or favorable functional profile have recently been suggested as hallmarks of a healthy gut microbiota (46). Certain probiotic strains may diminish the diversity of the gut microbiota either by production of 


\begin{tabular}{|c|c|c|c|c|c|}
\hline Phylum & Class & Order & Family & Genus & Species \\
\hline Firmicutes & Clostridia & Clostridiales & Ruminococcaceae & Oscillibacter' & unclassified \\
\hline Firmicutes & Clostridia & Clostridiales & Ruminococcaceae & unclassified & unclassified \\
\hline Fimicutes & Clostridia & Clostridiales & Ruminococcaceae & Butyricicoccus & unclassified \\
\hline Firmicutes & Clostridia & Clostridiales & Lachnospiracease & Coprococcus & unclassified \\
\hline Firmicutes & Clostridia & Clostridiales & unclassified & unclassified & unclassified \\
\hline Firmicutes & Clostridia & Clostridiales & Ruminococcaceae & unclassified & unclassified \\
\hline Firmicutes & Clostridia & Clostridiales & Lachnospiraceae & unclassified & unclassified \\
\hline Firmicutes & Clostridia & Clostridiales & Ruminococcaceae & unclassified & unclassified \\
\hline Firmicutes & Clostridia & Clostridiales & Lachnospiracease & unclassified & unclassified \\
\hline Firmicutes & Clostridia & Clostridiales & Ruminococcaceae & unclassified & unclassified \\
\hline Firmicutes & Clostridia & Clostridiales & Ruminococcaceze & unclassified & unclassified \\
\hline Firmicutes & Clostridia & Clostridiales & Ruminococcaceae & unclassified & unclassified \\
\hline Firmicutes & Clostridia & Clostridiales & Lachnospiracease & unclassified & unclassified \\
\hline Firmicutes & Clostridia & Clostridiales & Ruminococcaceae & unclassified & unclassified \\
\hline Firmicutes & Clostridia & Clostridiales & Ruminococcaceae & unclassified & unclassified \\
\hline Firmicutes & Clostridia & Clostridiales & Ruminococcaceae & unclassified & unclassified \\
\hline Firmicutes & Clostridia & Clostridiales & Lachnospiraceae & unclassified & unclassified \\
\hline Firmicutes & Clostridia & Clostridiales & Runinococcaceae & Oscillibacter & unclassified \\
\hline Bacteroidetes & s Bacteroidia & Bacteroidales & Porphyromonadaceas & atParabacteroides & unclassified \\
\hline Bacteroidetes & s Bacteroidia & Bacteroidales & Prevotellaceae & Prevotella & Prevotella copri \\
\hline Bacteroidetes & S Bacteroidia & Bacteroidales & Bacteroidacease & Bacteroides & unclassified \\
\hline Bacteroidetes & 5 Bacteroidia & Bacteroidales & Bacteroidacease & Bacteroides & unclassified \\
\hline Bacteroidetes & s Bacteroidia & Bacteroidales & Bacteroidacease & Bacteroides & Bacteroides ovatus \\
\hline Firmicutes & Clostridia & Clostridiales & Ruminococcaceae & Flavoniffactor & unclassified \\
\hline Firmicutes & Ensipelotichia & Erysipelotrichales & Envsipelotrichaceae & Clostridium XVIII & unclassified \\
\hline Bacteroidetes & s Bacteroidia & Bacteroidales & Bacteroidaceae & Bacteroides & unclassified \\
\hline Firmicutes & Negativicutes & Selenomonadales & Veilonellacese & Veillonella & unclassified \\
\hline Bacteroidetes & S Bacteroidia & Bacteroidales & Bacteroidacease & Bacteroides & unclassified \\
\hline Firmicutes & Clostridia & Clostridiales & Lachnospiraceae & Anaerostipes & unclassified \\
\hline Fusobacteria & Fusobacteriia & Fusobacteriales & Fusobacteriaceae & Fusobacterium & unclassified \\
\hline
\end{tabular}

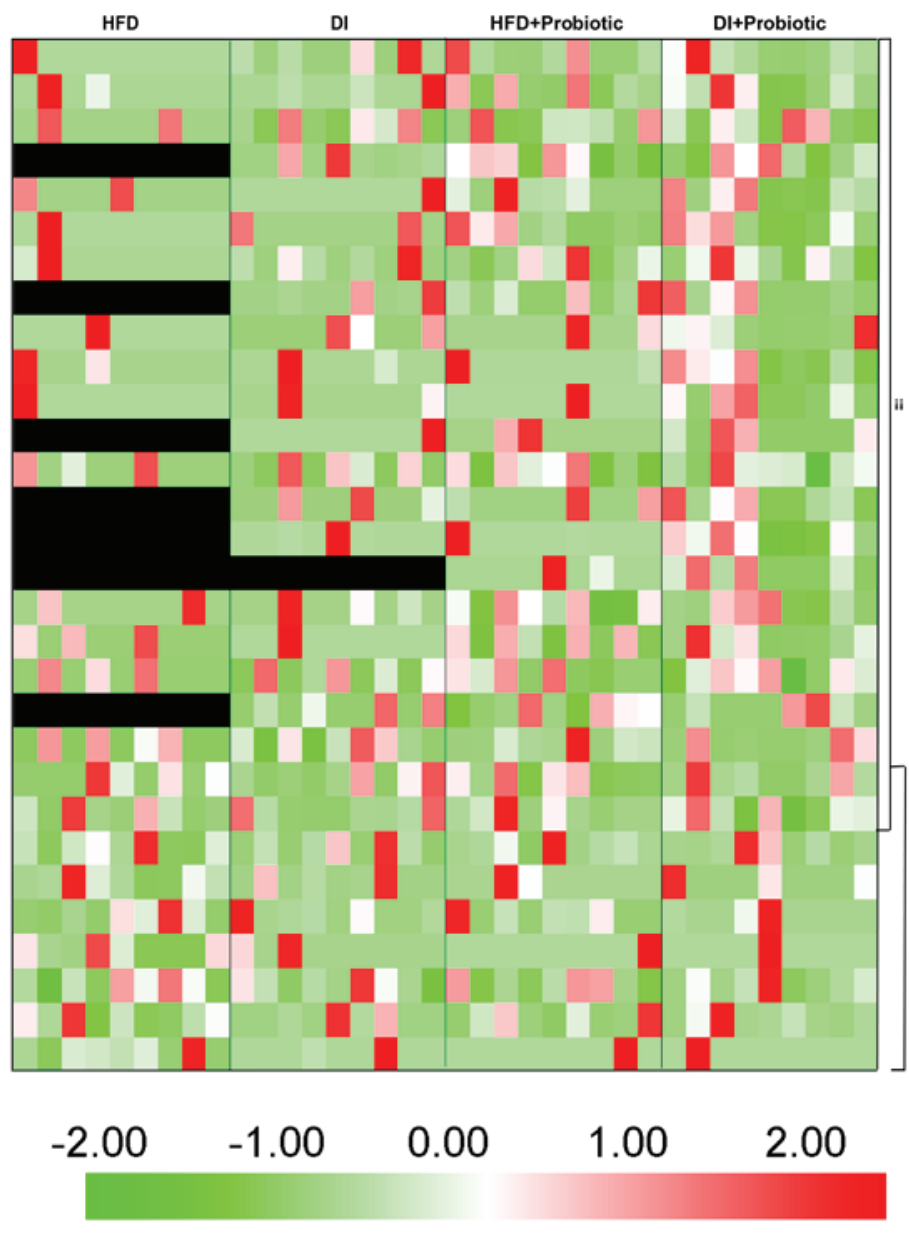

Row Z Score

Figure 4. Effect of dietary intervention and probiotic supplementation on the fecal microbiota composition at the species level. The relative abundance of altered species was visualized using a heatmap. Data are represented as row-scaled Z-scores. The black bars represent absent species. i and ii indicate species reduced and elevated in the DI, HFD + Probiotic and DI +Probiotic groups when respectively compared with the HFD group. Probiotics included Bifidobacterium longum, Lactobacillus acidophilus and Enterococcus faecalis. HFD, high-fat diet; DI, dietary intervention with low-fat diet.

anti-microbial peptides, or alternatively, by increasing competition for nutrients, processes that are able to reduce microbial growth. Hanifi et al (47) demonstrated that supplementation with Bacillus subtilis R0179 did not appear to reverse the overall microbiota diversity, as it simultaneously inhibited the growth of certain opportunistic pathogens. In the present study, it was observed that simply replacing an HFD with an LFD did not alter the $\alpha$-diversity of the fecal microbiota. A murine (RELM $\beta)$ knockout model study revealed that alterations in gut microbiome composition induced by dietary fat were independent of obesity (48). In the present study, it was revealed that $\sim 50 \%$ of all detected microbial species were shared by $44 \%$ of the volunteers, regardless of diet or treatment. However, divergent results have been identified in other studies. Intestinal microbiota diversity was reduced in HFDand high sugar diet-fed mice, whereas a control diet was able to prevent these changes. Heinsen et al (49) demonstrated that a very low-calorie diet beneficially altered the gut microbiome diversity in obese human subjects, but that these changes were not sustained during weight maintenance.

At the phylum level, the ratio of Bacteroidetes to Firmicutes was indicated to be decreased in diet-induced obesity, which could be reversibly increased by diet adaptation $(50,51)$.
By contrast, various studies have suggested that the ratio of Bacteroidetes to Firmicutes is not a contributing factor in human obesity, and it appears not to be associated with diet $(52,53)$. In line with this, in the present study, no significant differences were observed in the relative abundance of Bacteroidetes and Firmicutes among the four treatment groups, which may be due to differences in genetic background, age and sex. Furthermore, dietary intervention and probiotics supplementation were unable to change the bacterial composition at the phylum level, since Bacteroidetes and Firmicutes are the phyla predominantly present in the gut microbiota of humans (53-56).

At the species level, significant differences among the treatment groups were identified in the present study, which was consistent with the results of the animal study by Park et al (45). The relative abundance of 30 species was altered by dietary intervention and/or probiotic supplementation, 25 of which were changed in subjects who received dietary intervention and probiotic treatment. In addition, increased unclassified species mainly belonged to two butyrate-producing families, Ruminococcaceae and Lachnospiraceae (57), for which three taxa have been identified at the genus level, including Oscillibacter, Butyricicoccus and Coprococcus, which 
may be beneficial for colonic health (58-63). One study has indicated that two Lactobacillus strains, Lactobacillus plantarum HAC01 and L. rhamnosus GG, exert a beneficial effect on Lachnospiraceae and Ruminococcaceae at the bacterial family level, rather than Firmicutes and Bacteroidetes at the phylum level (64). The relative abundance of the Lachnospiraceae (phylum Firmicutes) was significantly higher in the Lactobacillus-treated groups compared with that in the PBS-treated control group. Amelioration of obesity-associated dysbiosis by alteration of the gut microbiota appears to be associated with 'indicator' bacterial taxa, including the family Lachnospiraceae. Among those eight species whose populations were decreased following probiotic supplementation and dietary intervention compared with the natural HFD group, one species identified was B. ovatus, which was considered to belong to a group of Bacteroides species sharing similar phenotypic characteristics to those of $B$. fragilis and $B$. vulgatus that were frequently identified in patients with IBDs, including Crohn's disease and ulcerative colitis $(65,66)$. To a certain extent, probiotic supplementation and dietary intervention may protect an HFD population from suffering from gastrointestinal inflammation disorders, and help to remold a healthy gut bacterial symbiosis through inhibition of IBD-associated specific bacterial taxa. Various bacterial families associated with unclassified species reduced by probiotic and dietary intervention have been identified as Clostridium XVIII and Anaerostipes. Certain evidence suggests that Clostridium XVIII may serve as the next 'smart' probiotics (67), and the genus Anaerostipes is associated with potential butyrate-producing bacteria (58). Why these beneficial bacteria are also reduced in number following probiotic intervention remains to be elucidated. A plausible hypothesis explaining this is that the potential of certain probiotic strains to 'rebalance' butyrate concentrations may protect the host under those physiological conditions associated with altered butyrate concentrations (58). One unknown species belonging to the genus Flavonifractor, which is capable of cleaving the flavonoid C-ring (68), was determined to decline after combined probiotic and dietary intervention. Depletion of a species belonging to Flavonifractor has been demonstrated in obese individuals (69) and another study suggested that treatment with Bifidobacterium catenulatum LI10 was able to attenuate this depletion (70), which was not in agreement with the results of the present study. Another study published by Toscano et al (71) indicated that, after one month of probiotic intake, a reduction in the population of Flavonifractor was observed, which was consistent with the result of the present study. In addition, other unknown species from the genera Veillonella and Fusobacterium, which may act as pathogens and carcinoma-associated taxa (71-77), were significantly reduced by probiotic and dietary intervention, suggesting an improvement of the gut bacterial community induced by an HFD. Of note, a higher abundance of the supplemented probiotic strains in fecal samples was not observed following supplementation of these probiotics, probably since they did not appear to colonize the intestine themselves within such a short study duration.

In conclusion, the present study revealed that the diversity of gut microbiota was promoted in HFD populations receiving probiotic treatment and dietary intervention, along with an increase in the populations of numerous beneficial species, and a reduction in the number of certain detrimental species. Taken together, the present results suggest that Lactobacillus acidophilus, Bifidobacterium longum and Enterococcus faecalis supplementation and dietary intervention may modulate the gut microbiota, and may provide a natural alternative to treat HFD-associated disorders.

\section{Acknowledgements}

Not applicable.

\section{Funding}

This work was supported by grants from the National Natural Science Foundation of China (grant. nos. 81472262 and81230057) and theEmerging Cutting-Edge Technology Joint Research Projects of Shanghai (grant. no. SHDC12012106).

\section{Availability of data and materials}

All data generated or analyzed during this study are included in this published article.

\section{Authors' contributions}

LMQ, RYG and HLQ designed the study; data collection and analysis was performed by LMQ, RYG and JMH; the manuscript was written by LMQ; and revisions of the manuscript were made by RYG and HLQ; All of the authors provided intellectual input for the study and approved the final version of the manuscript.

\section{Ethics approval and consent to participate}

All procedures performed involving human participants were in accordance with the ethical standards of the Institutional and National Research Committee and with the 1964 Helsinki Declaration and its later amendments or comparable ethical standards. The present study was approved by the Ethics Committee of the Institutional Review Boards of Shanghai Tenth People's Hospital (Shanghai, China) and Jiangyin People's Hospital (Jiangyin, China). Written informed consent was obtained from the patients or their guardians.

\section{Patient consent for publication}

Not applicable.

\section{Competing interests}

The authors declare that they have no competing interests.

\section{References}

1. Zoetendal EG, Rajilic-Stojanovic $M$ and de Vos WM: High-throughput diversity and functionality analysis of the gastrointestinal tract microbiota. Gut 57: 1605-1615, 2008.

2. Zoetendal EG, Raes J, van den Bogert B, Arumugam M, Booijink CC, Troost FJ, Bork P, Wels M, de Vos WM and Kleerebezem M: The human small intestinal microbiota is driven by rapid uptake and conversion of simple carbohydrates. ISME J 6: 1415-1426, 2012. 
3. Hooper LV, Midtvedt T and Gordon JI: How host-microbial interactions shape the nutrient environment of the mammalian intestine. Annu Rev Nutr 22: 283-307, 2002.

4. Backhed F, Ley RE, Sonnenburg JL, Peterson DA and Gordon JI: Host-bacterial mutualism in the human intestine. Science 307: 1915-1920, 2005.

5. Qin J, Li R, Raes J, Arumugam M, Burgdorf KS, Manichanh C, Nielsen T, Pons N, Levenez F, Yamada T, et al: A human gut microbial gene catalogue established by metagenomic sequencing. Nature 464: 59-65, 2010.

6. Lin CS, Chang CJ, Lu CC, Martel J, Ojcius DM, Ko YF, Young JD and Lai HC: Impact of the gut microbiota, prebiotics, and probiotics on human health and disease. Biomed J 37: 259-268, 2014

7. Brown K, DeCoffe D, Molcan E and Gibson DL: Diet-induced dysbiosis of the intestinal microbiota and the effects on immunity and disease. Nutrients 4: 1095-1119, 2012.

8. Abu-Shanab A and Quigley EM: The role of the gut microbiota in nonalcoholic fatty liver disease. Nat Rev Gastroenterol Hepatol 7: 691-701, 2010.

9. Chiu CC, Ching YH, Li YP, Liu JY, Huang YT, Huang YW, Yang SS, Huang WC and Chuang HL: Nonalcoholic fatty liver disease is exacerbated in High-Fat Diet-Fed gnotobiotic mice by colonization with the Gut Microbiota from patients with nonalcoholic steatohepatitis. Nutrients 9: E1220, 2017.

10. Federico A,Dallio M,Caprio GG, Ormando VM and Loguercio C: Gut microbiota and the liver. Minerva Gastroenterol Dietol 63: 385-398, 2017.

11. Budden KF, Gellatly SL, Wood DL, Cooper MA, Morrison M, Hugenholtz P and Hansbro PM: Emerging pathogenic links between microbiota and the gut-lung axis. Nat Rev Microbiol 15: 55-63, 2017.

12. Bercik P: The microbiota-gut-brain axis: Learning from intestinal bacteria? Gut 60: 288-289, 2011.

13. Collins SM and Bercik P: Gut microbiota: Intestinal bacteria influence brain activity in healthy humans. Nat Rev Gastroenterol Hepatol 10: 326-327, 2013.

14. Bienenstock J, Kunze W and Forsythe P: Microbiota and the gut-brain axis. Nutr Rev 73 (Suppl 1): S28-S31, 2015.

15. Dinan TG and Cryan JF: Gut-brain axis in 2016: Brain-gut-microbiota axis-mood, metabolism and behaviour. Nat Rev Gastroenterol Hepatol 14: 69-70, 2017.

16. Arumugam M, Raes J, Pelletier E, Le Paslier D, Yamada T, Mende DR, Fernandes GR, Tap J, Bruls T, Batto JM, et al: Enterotypes of the human gut microbiome. Nature 473: 174-180, 2011.

17. Wu GD, Chen J, Hoffmann C, Bittinger K, Chen YY, Keilbaugh SA, Bewtra M, Knights D, Walters WA, Knight R, et al Linking long-term dietary patterns with gut microbial enterotypes. Science 334: 105-108, 2011.

18. Bushman FD, Lewis JD and Wu GD: Diet, gut enterotypes and health: Is there a link? Nestle Nutr Inst Workshop Ser 77: 65-73, 2013.

19. Kim KA, Gu W, Lee IA, Joh EH and Kim DH: High fat diet-induced gut microbiota exacerbates inflammation and obesity in mice via the TLR4 signaling pathway. PLoS One 7: e47713, 2012.

20. David LA, Maurice CF, Carmody RN, Gootenberg DB, Button JE Wolfe BE, Ling AV, Devlin AS, Varma Y, Fischbach MA, et al: Diet rapidly and reproducibly alters the human gut microbiome. Nature 505: 559-563, 2014

21. Schulz MD, Atay C, Heringer J, Romrig FK, Schwitalla S, Aydin B, Ziegler PK, Varga J, Reindl W, Pommerenke C, et al: High-fat-diet-mediated dysbiosis promotes intestinal carcinogenesis independently of obesity. Nature 514: 508-512, 2014.

22. Ou JH, Ridlon JM, DeLany JP, Vipperla K, Newton K and O'Keefe SJ: Obesity and colon cancer risk: Is it the Fat? Gastroenterology 142: S313, 2012.

23. Gerritsen J, Smidt H, Rijkers GT and de Vos WM: Intestinal microbiota in human health and disease: The impact of probiotics. Genes Nutr 6: 209-240, 2011.

24. Hu X, Wang T, LiW, Jin F and Wang L: Effects of NS Lactobacillus strains on lipid metabolism of rats fed a high-cholesterol diet. Lipids Health Dis 12: 67, 2013.

25. Liboredo JC, Anastacio LR, Peluzio Mdo C, Valente FX, Penido LC, Nicoli JR and Correia MI: Effect of probiotics on the development of dimethylhydrazine-induced preneoplastic lesions in the mice colon. Acta Cir Bras 28: 367-372, 2013.

26. Bertkova I, Hijova E, Chmelarova A, Mojzisova G, Petrasova D, Strojny L, Bomba A and Zitnan R: The effect of probiotic microorganisms and bioactive compounds on chemically induced carcinogenesis in rats. Neoplasma 57: 422-428, 2010.
27. Wang W, Shi LP, Shi L and Xu L: Efficacy of probiotics on the treatment of non-alcoholic fatty liver disease. Zhonghua Nei Ke Za Zhi 57: 101-106, 2018 (In Chinese).

28. Fan YJ, Chen SJ, Yu YC, Si JM and Liu B: A probiotic treatment containing Lactobacillus, bifidobacterium and enterococcus improves IBS symptoms in an open label trial. J Zhejiang Univ Sci B 7: 987-991, 2006.

29. Yang Y, Xia Y, Chen H, Hong L, Feng J, Yang J, Yang Z, Shi C, $\mathrm{Wu} \mathrm{W}$, Gao R, et al: The effect of perioperative probiotics treatment for colorectal cancer: Short-term outcomes of a randomized controlled trial. Oncotarget 7: 8432-8440, 2016.

30. Liang S, Xu L, Zhang D and Wu Z: Effect of probiotics on small intestinal bacterial overgrowth in patients with gastric and colorectal cancer. Turk J Gastroenterol 27: 227-232, 2016.

31. Knights D, Ward TL, McKinlay CE, Miller H, Gonzalez A, McDonald D and Knight R: Rethinking 'enterotypes'. Cell Host Microbe 16: 433-437, 2014.

32. Xia F, Chen J, Fung WK and Li H: A logistic normal multinomial regression model for microbiome compositional data analysis. Biometrics 69: 1053-1063, 2013.

33. Qian L, Gao R, Hong L, Pan C, Li H, Huang J and Qin H: Association analysis of dietary habits with gut microbiota of a native Chinese community. Exp Ther Med 16: 856-866, 2018

34. Wan Y, Wang F, Yuan J, Li J, Jiang D, Zhang J, Huang T, Zheng J, Mann J and Li D: Effects of macronutrient distribution on weight and related cardiometabolic profile in healthy non-obese Chinese: A 6-month, randomized controlled-feeding trial. Ebiomedicine 22: 200-207, 2017.

35. Li W and Godzik A: Cd-hit: A fast program for clustering and comparing large sets of protein or nucleotide sequences. Bioinformatics 22: 1658-1659, 2006.

36. Deng W, Wang Y, Liu Z, Cheng H and Xue Y: HemI: A toolkit for illustrating heatmaps. PLoS One 9: e111988, 2014.

37. Weichselbaum E: Potential benefits of probiotics-main findings of an in-depth review. Br J Community Nurs 15: 110, 112, 114, 2010.

38. Iannitti T and Palmieri B: Therapeutical use of probiotic formulations in clinical practice. Clin Nutr 29: 701-725, 2010.

39. Gareau MG, Sherman PM and Walker WA: Probiotics and the gut microbiota in intestinal health and disease. Nat Rev Gastroenterol Hepatol 7: 503-514, 2010

40. Chan YK, Brar MS, Kirjavainen PV, Chen Y, Peng J, Li D, Leung FC and El-Nezami H: High fat diet induced atherosclerosis is accompanied with low colonic bacterial diversity and altered abundances that correlates with plaque size, plasma A-FABP and cholesterol: A pilot study of high fat diet and its intervention with Lactobacillus rhamnosus GG (LGG) or telmisartan in ApoE(-/-) mice. BMC Microbiol 16: 264, 2016.

41. Kato-Kataoka A, Nishida K, Takada M, Kawai M, Kikuchi-Hayakawa H, Suda K, Ishikawa H, Gondo Y, Shimizu K, Matsuki T, et al: Fermented milk containing Lactobacillus casei strain shirota preserves the diversity of the gut microbiota and relieves abdominal dysfunction in healthy medical students exposed to academic stress. Appl Environ Microbiol 82: 3649-3658, 2016

42. Lisko DJ, Johnston GP and Johnston CG: Effects of dietary yogurt on the healthy human gastrointestinal (GI) microbiome. Microorganisms 5: E6, 2017.

43. Kristensen NB, Bryrup T, Allin KH, Nielsen T, Hansen TH and Pedersen O: Alterations in fecal microbiota composition by probiotic supplementation in healthy adults: A systematic review of randomized controlled trials. Genome Med 8: 52, 2016.

44. Zhang J, Wang L, Guo Z, Sun Z, Gesudu Q, Kwok L, Menghebilige and Zhang H: 454 pyrosequencing reveals changes in the faecal microbiota of adults consuming Lactobacillus casei Zhang. FEMS Microbiol Ecol 88: 612-622, 2014.

45. Park DY, Ahn YT, Park SH, Huh CS, Yoo SR, Yu R, Sung MK, McGregor RA and Choi MS: Supplementation of Lactobacillus curvatus HY7601 and Lactobacillus plantarum KY1032 in diet-induced obese mice is associated with gut microbial changes and reduction in obesity. PLoS One 8: e59470, 2013.

46. Backhed F, Fraser CM, Ringel Y, Sanders ME, Sartor RB, Sherman PM, Versalovic J, Young V and Finlay BB: Defining a healthy human gut microbiome: Current concepts, future directions, and clinical applications. Cell Host Microbe 12: 611-622, 2012.

47. Hanifi A, Culpepper T, Mai V, Anand A, Ford AL, Ukhanova M, Christman M, Tompkins TA and Dahl WJ: Evaluation of bacillus subtilis R0179 on gastrointestinal viability and general wellness: A randomised, double-blind, placebo-controlled trial in healthy adults. Benef Microbes 6: 19-27, 2015 
48. Hildebrandt MA, Hoffmann C, Sherrill-Mix SA, Keilbaugh SA Hamady M, Chen YY, Knight R, Ahima RS, Bushman F and Wu GD: High-fat diet determines the composition of the murine gut microbiome independently of obesity. Gastroenterology 137: 1716-1724.e1-2, 2009.

49. Heinsen FA, Fangmann D, Muller N, Schulte DM, Ruehlemann MC, Turk K, Settgast U, Lieb W, Baines JF, Schreiber S, et al: Beneficial effects of a dietary weight loss intervention on human gut microbiome diversity and metabolism are not sustained during weight maintenance. Obes Facts 9: 379-391, 2016.

50. Bell DS: Changes seen in gut bacteria content and distribution with obesity: Causation or association? Postgrad Med 127: 863-868, 2015

51. Shang Y, Khafipour E, Derakhshani H, Sarna LK, Woo CW, Siow YL and $\mathrm{O}$ K: Short term high fat diet induces Obesity-Enhancing changes in mouse gut microbiota that are partially reversed by cessation of the high fat diet. Lipids 52: 499-511, 2017.

52. Schwiertz A, Taras D, Schafer K, Beijer S, Bos NA, Donus C and Hardt PD: Microbiota and SCFA in lean and overweight healthy subjects. Obesity (Silver Spring) 18: 190-195, 2010.

53. Duncan SH, Lobley GE, Holtrop G, Ince J, Johnstone AM, Louis P and Flint HJ: Human colonic microbiota associated with diet, obesity and weight loss. Int J Obes (Lond) 32: 1720-1724, 2008.

54. Human Microbiome Project Consortium: Structure, function and diversity of the healthy human microbiome. Nature 486: 207-214, 2012.

55. Zhang J, Guo Z, Xue Z, Sun Z, Zhang M, Wang L, Wang G, Wang F, Xu J, Cao $\mathrm{H}$, et al: A phylo-functional core of gut microbiota in healthy young Chinese cohorts across lifestyles, geography and ethnicities. ISME J 9: 1979-1990, 2015.

56. Mariat D, Firmesse O, Levenez F, Guimaraes V, Sokol H, Dore J, Corthier G and Furet JP: The Firmicutes/Bacteroidetes ratio of the human microbiota changes with age. BMC Microbiol 9: 123, 2009.

57. Kang C, Wang B, Kaliannan K, Wang X, Lang H, Hui S, Huang L, Zhang Y, Zhou M, Chen M and Mi M: Gut microbiota mediates the protective effects of dietary capsaicin against chronic low-grade inflammation and associated obesity induced by high-fat diet. MBio 8: e00470-e00417, 2017.

58. Ferrario C, Taverniti V, Milani C, Fiore W, Laureati M, De Noni I, Stuknyte M, Chouaia B, Riso P and Guglielmetti S: Modulation of fecal clostridiales bacteria and butyrate by probiotic intervention with Lactobacillus paracasei DG varies among healthy adults. J Nutr 144: 1787-1796, 2014

59. Song JJ, Tian WJ, Kwok LY, Wang YL, Shang YN, Menghe B and Wang JG: Effects of microencapsulated Lactobacillus plantarum LIP-1 on the gut microbiota of hyperlipidaemic rats. Brit J Nutr 118: 481-492, 2017.

60. Eeckhaut V, Machiels K, Perrier C, Romero C, Maes S, Flahou B, Steppe M, Haesebrouck F, Sas B, Ducatelle R, et al: Butyricicoccus pullicaecorum in inflammatory bowel disease. Gut 62: 1745-1752, 2013.

61. Devriese S, Eeckhaut V, Geirnaert A, Van den Bossche L, Hindryckx P, Van de Wiele T, Van Immerseel F, Ducatelle R, De Vos M and Laukens D: Reduced Mucosa-associated butyricicoccus activity in patients with ulcerative colitis correlates with aberrant claudin-1 expression. J Crohns Colitis 11: 229-236, 2017.

62. Walker AW, Ince J, Duncan SH, Webster LM, Holtrop G, Ze X, Brown D, Stares MD, Scott P, Bergerat A, et al: Dominant and diet-responsive groups of bacteria within the human colonic microbiota. ISME J 5: 220-230, 2011.
63. An C, Kuda T, Yazaki T, Takahashi H and Kimura B: Caecal fermentation, putrefaction and microbiotas in rats fed milk casein, soy protein or fish meal. Appl Microbiol Biotechnol 98: 2779-2787, 2014

64. Park S, Ji Y, Jung HY, Park H, Kang J, Choi SH, Shin H, Hyun CK, Kim KT and Holzapfel WH: Lactobacillus plantarum HAC01 regulates gut microbiota and adipose tissue accumulation in a diet-induced obesity murine model. Appl Microbiol Biotechnol 101: 1605-1614, 2017.

65. Saitoh S, Noda S, Aiba Y, Takagi A, Sakamoto M, Benno Y and Koga Y: Bacteroides ovatus as the predominant commensal intestinal microbe causing a systemic antibody response in inflammatory bowel disease. Clin Diagn Lab Immunol 9: 54-59, 2002.

66. Dicksved J, Halfvarson J, Rosenquist M, Jarnerot G, Tysk C, Apajalahti J, Engstrand L and Jansson JK: Molecular analysis of the gut microbiota of identical twins with Crohn's disease. ISME J 2: 716-727, 2008.

67. El Hage R, Hernandez-Sanabria E and Van de Wiele T: Emerging trends in 'Smart Probiotics': Functional consideration for the development of novel health and industrial applications. Front Microbiol 8: 1889, 2017.

68. Winter J, Moore LH, Dowell VR Jr and Bokkenheuser VD: C-ring cleavage of flavonoids by human intestinal bacteria. Appl Environ Microbiol 55: 1203-1208, 1989.

69. Kasai C, Sugimoto K, Moritani I, Tanaka J, Oya Y, Inoue H, Tameda M, Shiraki K, Ito M, Takei Y and Takase K: Comparison of the gut microbiota composition between obese and non-obese individuals in a Japanese population, as analyzed by terminal restriction fragment length polymorphism and next-generation sequencing. BMC Gastroenterol 15: 100, 2015.

70. Fang D, Shi D, Lv L, Gu S, Wu W, Chen Y, Chen Y, Guo J, Li A, Xinjun $\mathrm{Hu}$, et al: Bifidobacterium pseudocatenulatum LI09 and Bifidobacterium catenulatum LI10 attenuate D-galactosamine-induced liver injury by modifying the gut microbiota. Sci Rep 7: 8770, 2017.

71. Toscano M, De Grandi R, Miniello VL, Mattina R and Drago L: Ability of Lactobacillus kefiri LKF01 (DSM32079) to colonize the intestinal environment and modify the gut microbiota composition of healthy individuals. Dig Liver Dis 49: 261-267, 2017.

72. Jimenez MB: Treatment of irritable bowel syndrome with probiotics. An etiopathogenic approach at last? Rev Esp Enferm Dig 101: 553-564, 2009

73. van den Bogert B, Meijerink M, Zoetendal EG, Wells JM and Kleerebezem M: Immunomodulatory properties of Streptococcus and Veillonella isolates from the human small intestine microbiota. PLoS One 9: e114277, 2014.

74. Garrido D, Suau A, Pochart P, Cruchet S and Gotteland M: Modulation of the fecal microbiota by the intake of a Lactobacillus johnsonii La1-containing product in human volunteers. FEMS Microbiology Letters 248: 249-256, 2005.

75. Zhu Q, Jin Z, Wu W, Gao R, Guo B, Gao Z, Yang Y and Qin H: Analysis of the intestinal lumen microbiota in an animal model of colorectal cancer. PLoS One 9: e90849, 2014.

76. Hibberd AA, Lyra A, Ouwehand AC, Rolny P, Lindegren H, Cedgard $\mathrm{L}$ and Wettergren $\mathrm{Y}$ : Intestinal microbiota is altered in patients with colon cancer and modified by probiotic intervention. BMJ Open Gastroenterol 4: e000145, 2017.

77. Wong SH, Kwong TNY, Chow TC, Luk AKC, Dai RZW, Nakatsu G, Lam TYT, Zhang L, Wu JCY, Chan FKL, et al: Quantitation of faecal Fusobacterium improves faecal immunochemical test in detecting advanced colorectal neoplasia. Gut 66 : $1441-1418,2017$. 